\title{
Flow of hypermagnetic helicity in the embryo of a new phase in the electroweak phase transition
}

\author{
P.M. Akhmet'ev,, , 田 V.B. Semikoz,, , 田 and D.D. Sokoloff ${ }^{2, \text { 团 }}$ \\ ${ }^{1}$ Pushkov Institute of Terrestrial Magnetism, \\ Ionosphere and Radiowave Propagation of the Russian Academy of Sciences, \\ IZMIRAN, Troitsk, Moscow region, 142190, Russia \\ ${ }^{2}$ Department of Physics, Moscow State University, 119999, Moscow, Russia
}

(Dated: November 13, 2018)

\begin{abstract}
The dynamics of the magnetic helicity during the electroweak phase transition in the early Universe is studied. It is shown that the boundary surface between symmetric (hypermagnetic) phase and Maxwellian phase with a broken symmetry is a membrana for the separation of the magnetic helicity. Assuming the total linking number of knots of hypermagnetic field is negative, it is proved that the helicity rising in the Maxwellian phase is left-handed.

PACS numbers: 14.60.-z 13.15.+g 95.30.Qd 52.30.Cv
\end{abstract}

The primordial magnetic fields in the early Universe could be sufficiently strong not influencing neither expansion of universe nor Big Bang nucleosynthesis. If such fields survive under certain conditions after recombination $(z<1100)$ they could be as seed fields in the galactic dynamo mechanism [1, 2].

An essential topological characteristic of the magnetic field which is global invariant in expanding universe with the changing structure of magnetic field at different scales is the magnetic helicity, for example, $H=\int d^{3} x(\mathbf{B} \cdot \mathbf{A})$ in the case of the Maxwellian field. By a modern knowledge [3], it can significantly affect the evolution of magnetic fields in galaxies.

The cosmological magnetic field and its helicity can be formed as a result of phase transitions in the early Universe and, in particular, in the electroweak phase transition. In this phase transition the hypermagnetic field converts into the Maxwellian electromagnetic field.

In this paper we try to study how the helicity of hypermagnetic fields is related to the magnetic helicity of Maxwellian fields during electroweak phase transition. We show that during this phase transition on the surface separating the phases a separation of magnetic helicity exists. The magnetic helicity being collected in Maxwellian phase in the course of this separation is conserved then in further expansion of the Universe and the subsequent formation of galaxies. As shown in [4, 5], the phenomenon is due to neutrino asymmetry and parity violation $(P$-non-invariance) in weak interactions. In the absence of neutrino asymmetry in an ideal plasma the helicity is conserved.

Let us consider a bubble (an embryo of the Maxwell phase) of the radius $R$, inside of the hot plasma in the

\footnotetext{
*Electronic address: pmakhmet@izmiran.ru

$\dagger$ Electronic address: semikoz@yandex.ru

${ }_{\ddagger}^{\ddagger}$ Electronic address: sokoloff@dds.srcc.msu.su
}

early Universe at the moment of the electroweak phase transition with the temperature $T_{E W} \sim 100 \mathrm{GeV}$. Let us assume that this bubble is growing with the constant velocity, $R(t)=v\left(t-t_{E W}\right)$, where the velocity $v$ itself $(v=0.1-1$ according to [6] ) is unessential (cancelled) in the solution to our problem.

It is important for our calculation that the value $\left(t-t_{E W}\right) / t_{E W} \ll 1$ is small, or that the temperature during the phase transition remains constant at the moment $t_{E W}=M_{0} / 2 T_{E W}^{2}=0.23 \times 10^{-10} \mathrm{c}$, where $M_{0}=M_{P l} / 1.66 \sqrt{g^{*}}$ is given by the Plank mass $M_{P l}=1.2 \times 10^{19} \mathrm{GeV}$ and by the degree of freedom $g^{*} \sim 100$. This implies that the radius of the bubble is much less than the horizon size $\left(2 t_{E W}=l_{H}=1.44 \mathrm{~cm}\right)$, $R \ll l_{H}$. More precisely, we shall assume that the radius of the bubble is much less than the scale of the mean hypermagnetic field, $R \ll \eta_{Y} / \alpha_{Y} \ll l_{H}$.

In the rest frame of the medium as a whole the induction equation governing hypermagnetic fields $\mathbf{B}_{Y}=$ $\nabla \times \mathbf{Y}$ outside of the bubble reads:

$$
\frac{\partial \mathbf{B}_{Y}}{\partial t}=\nabla \times \alpha_{Y} \mathbf{B}_{Y}+\eta_{Y} \nabla^{2} \mathbf{B}_{Y}
$$

while the evolution equation for Maxwellian field $\mathbf{B}=$ $\nabla \times \mathbf{A}$ within the bubble is analogous, but with different value of the parameter $\alpha$. This parameter characterizes $P$-non-invariance of weak interactions and it is a scalar, but not a pseudoscalar like the corresponding parameter $<\mathbf{v} \cdot(\nabla \times \mathbf{v})>$ in the standard magnetohydrodynamics [3].

After the phase transition such a parameter of the helicity is the following [4]:

$$
\alpha=2 \times 10^{-2} G_{F} T \lambda^{-1} \sum_{a} c_{a}^{(A)} \xi_{\nu_{a}},
$$

while before the phase transition it takes the form [5]:

$$
\alpha_{Y}=3 \times 10^{-3} g^{\prime 2} \sigma^{-1} T \sum_{a} \xi_{\nu_{a}},
$$


where $G_{F}$ is the Fermi constant, $T$ is the temperature, $\lambda$ is the spatial parameter of the inhomogeneity of the neutrino gas; $c_{a}^{(A)}=\mp 0.5$ is the axial constant of weak interactions (the upper sign for electron neutrino); $g^{\prime}$ is the coupling constant for hypercharge field in the WeinbergSalam model; $\xi_{\nu_{a}}=\mu_{\nu_{a}} / T$ is the dimensionless chemical potential of neutrino, $a=e, \mu, \tau$, and coefficients of hypermagnetic diffusion $\eta=\eta_{Y}=(4 \pi \sigma)^{-1}$ are given by the conductivity of plasma $\sigma \sim 100 T$ and, practically, coincide for both phases. All coefficients $\xi_{\nu_{a}}, \alpha_{Y}, \eta_{Y}$ depend on the temperature (or time) by the Friedman law, but for given problem at the fixed temperature of the phase transition $T_{E W}$, these parameters remain unaltered.

Multiplying Eq. (11) and its analogue for Maxwellian field by the corresponding vector potential and adding the analogous construction produced by evolution equation governing the vector potential (multiplied by hypermagnetic or magnetic field) after the integration over the space we get the evolution equation for the total helicity $H=\int(\mathbf{B} \cdot \mathbf{A}) d^{3} x+\int\left(\mathbf{B}_{Y} \cdot \mathbf{Y}\right) d^{3} x$, where the integration is carried over the domains with Maxwell phase and hypermagnetic field correspondingly. This equation takes the form:

$$
\begin{aligned}
\frac{\mathrm{d} H}{\mathrm{dt}}= & -2 \int(\mathbf{E} \cdot \mathbf{B}) \mathrm{d}^{3} x- \\
& -\oint_{S}\left(\left(\mathbf{E} \times \mathbf{A}+A_{0} \mathbf{B}\right) \cdot \mathbf{n}\right) \mathrm{d}^{2} S+\ldots,
\end{aligned}
$$

where the dots mean analogous terms for hypermagnetic field. We take into account the surface integrals which are omitted in problems for a monophase medium [7] as integrals over an infinite boundary of the domain. In our problem namely these integrals determine the flow of the helicity through the boundary of a bubble of the radius $R$, on which a separation of the helicity takes place. Accounting for the boundary condition $A_{\mu}=\cos \theta_{W} Y_{\mu}$, where $\sin ^{2} \theta_{W}=0.23$ is the parameter of the standard Weinberg-Salam model, the integrals above are calculated over the surface as following:

$$
\frac{\mathrm{d} H_{Y}}{\mathrm{dt}}=-\sin ^{2} \theta_{W} \oint\left(\mathbf{E}_{Y} \times \mathbf{Y}+Y_{0} \mathbf{B}_{Y}\right) \mathbf{n}_{Y} \mathrm{~d}^{2} S,
$$

where the unit normal vector $\mathbf{n}_{Y}=-\hat{\mathbf{e}}_{r}=(-1,0,0)$ is directed inwards the bubble with Maxwellian phase (the phase with broken symmetry).

The flow of hypermagnetic helicity density, penetrated inside the bubble through the surface at the moment of electroweak phase transition, is the pseudovector given by the formula

$$
\mathbf{S}=\mathbf{n}_{Y} h_{Y}(t)=\mathbf{n}_{Y}\left(\frac{1}{4 \pi R^{2}(t) d}\right) \int_{t_{E W}}^{t} \mathrm{dt} \frac{\mathrm{d} H_{Y}(t)}{\mathrm{dt}} .
$$

This flow is analogous to the vector flow of the energy of a flat electromagnetic wave $\mathbf{S}=W \mathbf{n}$, where $W=$
$\left(E^{2}+B^{2}\right) / 8 \pi$ is the energy density of the field. Here $4 \pi d R^{2}(t)$ is the volume of a thin spherical layer with the thickness $d$ of the domain wall separating the two phases. For fields at the scale $R, Y \sim B_{Y} R$, the value of the flow is inversely to the thickness $d, h_{Y} \sim d^{-1}$.

It is not difficult to prove that the surface integrals are equal to zero, i.e. there is no separation of the helicity if we substitute the flat hypermagnetic field, $Y_{0}=Y_{z}=0$, $Y_{x}=Y(t) \sin k_{0} z, Y_{y}=Y(t) \cos k_{0} z$, (see estimates of the baryon asymmetry in [8]). But in the case of 3Dfield with nonzero helicity the considered integrals are nontrivial. Let us consider the following potential of the hypermagnetic field with the number of the linked loops equal to $n$ :

$$
\begin{aligned}
Y_{r}(t, \rho, \theta)= & \frac{-Y(t) \cos \theta}{\left(\rho^{2}+1\right)^{2}} \\
Y_{\theta}(t, \rho, \theta)= & \frac{Y(t) \sin \theta}{\left(\rho^{2}+1\right)^{2}}\left[1+B(\rho-1)^{2}+b(\rho-1)^{3}\right] \\
Y_{\phi}(t, \rho, \theta)= & \frac{-Y(t) n \sin \theta}{\left(\rho^{2}+1\right)^{2}}\left[\rho+C(\rho-1)^{2}+\right. \\
& \left.+(C+c)(\rho-1)^{3}\right]
\end{aligned}
$$

where $\rho=r / R$, dependence on time is $Y(t)=2 B_{0}(t) / \pi R$ (compare [10]), and the coefficients $b, c, B, C$ are calculated below. We correct here the misprint in our paper [9] where in the same Eq. (77) we missed the amplitude $Y(t)$ for the component $Y_{\theta}(t, \rho, \theta)$.

The hypermagnetic field $\mathbf{B}_{Y}=\nabla \times \mathbf{Y}$ near the surface of phase separation, $0<\rho-1 \ll 1$, has the components

$$
\begin{aligned}
& B_{r}^{Y}=\frac{1}{r \sin \theta}\left[\frac{\partial}{\partial \theta}(\sin \theta) Y_{\phi}\right]=-\frac{Y(t) n \cos \theta}{2 R(t)} \times \\
& \times\left[1-2(\rho-1)+(C+2)(\rho-1)^{2}+O\left((\rho-1)^{3}\right)\right] \\
& B_{\theta}^{Y}=-\frac{1}{r}\left[\frac{\partial}{\partial r}\left(r Y_{\phi}\right)\right]=\frac{Y(t) n \sin \theta}{2 R(t)}[(\rho-1)(C-1) \\
& \left.+(\rho-1)^{2}\left(\frac{3}{2} c+\frac{5}{2}-C\right)+O\left((\rho-1)^{3}\right)\right] \\
& B_{\phi}^{Y}=\frac{1}{r}\left[\frac{\partial}{\partial r}\left(r Y_{\theta}\right)-\frac{\partial Y_{r}}{\partial \theta}\right]=\frac{Y(t) \sin \theta}{4 R(t)}[-2+ \\
& +(\rho-1)(4+2 B)+(\rho-1)^{2}(3 b-3-5 B)+ \\
& \left.+O\left((\rho-1)^{3}\right)\right] .
\end{aligned}
$$

At the surface of bubble $\rho=1$ our potential (7) and corresponding hypermagnetic field (8) are like in paper 10]. Obviously, $\nabla \cdot \mathbf{B}_{Y}=0$. We used the Lorentz gauge $\partial Y_{\mu} / \partial x_{\mu}=0$ to calculate the temporal component of hypercharge field

$$
Y_{0}(\rho, \theta, t)=-\frac{4 \rho \cos \theta}{\left(\rho^{2}+1\right)^{3}} \int_{t_{E W}}^{t} \frac{Y\left(t^{\prime}\right)}{R\left(t^{\prime}\right)} \mathrm{d} t^{\prime}
$$


A straightforward calculation of the surface term (5) gives the following equation:

$$
\frac{\mathrm{d} H_{Y}(t)}{\mathrm{dt}}=\frac{2 \pi \sin ^{2} \theta_{W} n}{3} R(t) Y(t) \int_{t_{E W}}^{t} \frac{Y\left(t^{\prime}\right)}{R\left(t^{\prime}\right)} \mathrm{d} t^{\prime},
$$

where we substituted in the expression $\mathbf{E}_{Y}=-\partial \mathbf{Y} / \partial t-$ $\nabla Y_{0}$ the gradient $\nabla Y_{0}$

$$
\begin{aligned}
\nabla Y_{0}= & \frac{1}{R(t)} \int_{t_{E W}}^{t} \frac{Y\left(t^{\prime}\right) \mathrm{d} t^{\prime}}{R\left(t^{\prime}\right)}\left[\frac{4 \sin \theta \hat{\mathbf{e}}_{\theta}}{\left(\rho^{2}+1\right)^{3}}-\right. \\
& \left.-\frac{4 \cos \theta\left(1-5 \rho^{2}\right) \hat{\mathbf{e}}_{r}}{\left(\rho^{2}+1\right)^{4}}\right]
\end{aligned}
$$

and took into account that in the case of the axialsymmetric configuration (7) the vector $B_{r}^{Y}$ is independent of the coordinate $\phi$. The values in Eq. (9) including $\nabla Y_{0} \times \mathbf{Y}=\hat{\mathbf{e}}_{r}\left(\nabla Y_{0}\right)_{\theta} Y_{\phi}$ are calculated at the surface of bubble $\rho=1$.

Hence the problem is reduced to the calculation $Y(t)$ from the Faraday equation (11) which for the considered potential (7) and hypermagnetic field (8) at the boundary $\rho=1$ can be rewritten by components as

$$
\begin{aligned}
& \frac{\partial B_{r}^{Y}}{\partial t}-\eta_{Y}\left(\nabla^{2} \mathbf{B}_{Y}\right)_{r}=\frac{\alpha_{Y}}{r \sin \theta} \frac{\partial}{\partial \theta}\left(\sin \theta B_{\phi}^{Y}\right)= \\
& =-\frac{\alpha_{Y} Y(t) \cos \theta}{R^{2}(t)} \\
& \frac{\partial B_{\theta}^{Y}}{\partial t}-\eta_{Y}\left(\nabla^{2} \mathbf{B}_{Y}\right)_{\theta}=-\frac{\alpha_{Y}}{r} \frac{\partial}{\partial r}\left(r B_{\phi}^{Y}\right)= \\
& =-\frac{\alpha_{Y} Y(t) \sin \theta}{2 R^{2}(t)}(B+1)=0 \\
& \frac{\partial B_{\phi}^{Y}}{\partial t}-\eta_{Y}\left(\nabla^{2} \mathbf{B}_{Y}\right)_{\phi}=\frac{\alpha_{Y}}{r}\left[\frac{\partial}{\partial r}\left(r B_{\theta}^{Y}\right)-\frac{\partial B_{r}^{Y}}{\partial \theta}\right]= \\
& =\frac{\alpha_{Y} Y(t) n \sin \theta}{2 R^{2}(t)}(C-2)
\end{aligned}
$$

Let us pay attention to the zeroth result for $B_{\theta}^{Y}$ at the boundary $\rho=1$ (see also in Eq. (8)) that forces us to choose $B=-1$ in Eq. (11)) for that component. The Laplacian in the 1.h.s. of Faraday equation for the same component also vanishes at the boundary $\rho=1$, $\left(\nabla^{2} \mathbf{B}_{Y}\right)_{\theta}=(5+3 c) Y(t) n \sin \theta / 2 R^{3}(t)=0$, if we choose $c=-5 / 3$.

Then accounting for the other Laplacian components on the same boundary surface, the radial one, $\left(\nabla^{2} \mathbf{B}_{Y}\right)_{r}=Y(t) n \cos \theta(2-C) / R^{3}(t) \neq 0$, and the zeroth (under conditions $b=-2, B=-1$ ) azimuthal component, $\left(\nabla^{2} \mathbf{B}_{Y}\right)_{\phi}=Y(t) \sin \theta(3 b-3 B+3) / 2 R^{3}(t)=0$, one gets from the first equation (11) for radial component,

$$
\frac{\dot{Y}}{Y}-\frac{\dot{R}}{R}=\frac{2 \alpha_{Y}}{n R}-\frac{\eta_{Y}(4-2 C)}{R^{2}}
$$

while from the third equation (11) for the azimuthal component we find

$$
\frac{\dot{Y}}{Y}-\frac{\dot{R}}{R}=\frac{\alpha_{Y} n(2-C)}{R} .
$$

Thus, in addition to the parameters $B=-1, c=-5 / 3$, $b=-2$ combining eqs. (12) and (13) we find the last parameter $C(t)$ in Eq. (7),

$$
C(t)=\frac{2\left(n-n^{-1}\right) \alpha_{Y} R^{-1}+4 \eta_{Y} R^{-2}}{n \alpha_{Y} R^{-1}+2 \eta_{Y} R^{-2}} .
$$

Substituting the parameter (14), e.g., into Eq. (13) one obtains the ordinary differential equation for the amplitude $Y(t)$,

$$
\frac{\dot{Y}(t)}{Y(t)}-\frac{\dot{R}(t)}{R(t)}=\frac{2 \alpha_{Y}^{2}}{n \alpha_{Y} R(t)+2 \eta_{Y}} .
$$

In the realistic situation of finite conductivity a scale of the mean hypermagnetic field $\Lambda=\kappa \eta_{Y} / \alpha_{Y}$, where $\kappa \geq 1$, should be much bigger than the diameter of the bubble in the new phase, i.e. the following inequality has to be satisfied: $\alpha_{Y} R(t) \ll \kappa \eta_{Y}$. If a more stronger condition $\alpha_{Y} R(t) \ll 2 \eta_{Y} / n \leq \kappa \eta_{Y}$ is fulfilled, then from (15) for the function $B_{Y}(t)=Y(t) / R(t)$ using (3) we get

$$
\begin{aligned}
& B_{Y}(t)=B_{Y}\left(t_{E W}\right) \exp \left[\left(\frac{\alpha_{Y}^{2}}{\eta_{Y}}\right)\left(t-t_{E W}\right)\right]= \\
& =B_{Y}\left(t_{E W}\right) \exp \left[63\left(\frac{\xi_{\nu}}{0.001}\right)^{2} \frac{\left(t-t_{E W}\right)}{t_{E W}}\right],
\end{aligned}
$$

where $B_{Y}\left(t_{E W}\right)$ is the hypermagnetic field amplitude on the scale of the bubble, $\alpha_{Y}=\alpha_{Y}\left(T_{E W}\right), \eta_{Y}=$ $\eta_{Y}\left(T_{E W}\right)$ are the constant coefficients at the moment of the phase transition, the sum $\xi_{\nu}=\sum_{a} \xi_{\nu_{a}}\left(T_{E W}\right)$ is the net neutrino asymmetry (neutrino degeneracy parameter); $\left(t-t_{E W}\right) / t_{R W} \ll 1$ is a small parameter for selfconsistency of our problem (see above).

Substituting the amplitude of the hypercharge field $Y(t)=B_{Y}(t) R(t)$ on the surface of the phase separation (16) into the expression of the surface integral (9), after the integration over time and division by the volume of the spherical layer with the thickness $d$ we get from (6) the value of the flow of hypermagnetic helicity density through the surface of the bubble,

$$
\frac{h_{Y}(t)}{G^{2} c m}=\frac{5 \times 10^{-3} n}{d(c m)}\left(\frac{B_{Y}\left(t_{E W}\right)}{1 G}\right)^{2}\left(\frac{t-t_{E W}}{t_{E W}}\right)^{2}
$$

An unknown neutrino asymmetry at the moment of the phase transition is estimated by $\left(\xi_{\nu} / 0.001\right) \simeq 0.12$. This estimate corresponds to the restrictions in Eq. (24) in paper [5], obtained from the condition that the hypermagnetic field survives against ohmic diffusion for spatial scales $\sim \eta_{Y} / \alpha_{Y}$. 
Let us note that in order to avoid the screening of the hyperelectric field $\mathbf{E}_{Y}$ and the temporal component $Y_{0}$ over the surface of the bubble, the thickness $d$ of the domain wall should be less than the Debye radius, $d<r_{D}=\sqrt{3 T_{E W} / 4 \pi e^{2} n_{e}} \sim 10 / T_{E W}$, that allows to estimate the factor $d^{-1}$ in the formula (17) as $d^{-1}(\mathrm{~cm})>10^{15} / 2$. This means that a moderate hypermagnetic field $B_{Y}\left(t_{E W}\right)$ provides a huge flow of the helicity density (17).

Indeed, substituting into (17) the value of hypermagnetic field at the moment of phase transition $B_{Y}\left(t_{E W}\right)$ estimated in [8] as $B_{Y}\left(t_{E W}\right) \sim 5 \times 10^{17} \mathrm{G}$, one gets $h / G^{2} \mathrm{~cm}>6.25 \times 10^{47}\left[\left(t-t_{E W}\right) / t_{E W}\right]^{2}$. Such huge value estimated at the moment of the growth of a bubble of the new phase, e.g, for $R(t) / l_{H}<\left[\left(t-t_{E W}\right) / t_{E W}\right] \sim 10^{-6}$, accounting for the following conservation of the net global helicity summed over different protogalactic scales, occurs much bigger than the helicity density of galactic magnetic field $h_{\text {gal }} \sim 10^{11} \mathrm{G}^{2} \mathrm{~cm}$, (see also estimates of the primordial magnetic helicity in paper [11]).

We have to note that a growth of hypermagnetic field before the electroweak phase transition depends essentially (exponentially) on the neutrino asymmetry $\left(B_{Y}(t)=B_{0}^{Y} \exp \left[\int_{t_{0}}^{t}\left(\alpha_{Y}^{2}\left(t^{\prime}\right) / 4 \eta_{Y}\left(t^{\prime}\right)\right) d t^{\prime}\right]\right.$ in $\alpha^{2}$-dynamo [5]). But in the expression for helicity (17) a hypermagnetic field $B_{Y}(t) \approx B_{Y}\left(t_{E W}\right)$ is fixed at the moment of the phase transition, moreover, for a small bubble the answer is practically independent of neutrino asymmetry in the time-depended field $B_{Y}(t)$ given by (16).

The single bubble of the Maxwellian phase inside of ambient symmetric phase with the potential given by Eq. (7) near the boundary, is a reasonable approximation during the beginning of the phase transition before percolation (junction of bubbles). One can consider also another final step of the phase transition, when a new phase with broken symmetry prevails and a single bubble of the symmetric phase with hypermagnetic field inside exists. It is not hard to check that in this case the change of sign $\rho-1>0$ to $\rho-1<0$ in the potential (7) gives the same components of hypermagnetic field inside the bubble $\rho<1$. Let us note that in the considered approximation (7) magnetic charges near the surface of the phase transition and over this surface itself are absent, $\nabla \cdot \mathbf{B}_{Y}=0$.

A choice of the negative sign of the helicity density (17) if $n<0$ corresponds to the result [12] for the lefthanded magnetic helicity in the same electroweak phase transition. That result is obtained for the mechanism of decay of linked loops of $Z$-strings leading to creation of magnetic monopole-antimonopole pairs at the ends of a decaying string, after which the reconnection of each such pair proceeds through junction by loops of Maxwellian field.

Let us recall that the pseudoscalar $n$ is the number of pairs of linking magnetic field loops entering the Gauss integral for magnetic helicity, $H(t)=\int d^{3} x h(t, \mathbf{x})=$ $n \Phi_{1} \Phi_{2}[7]$. This pseudoscalar changes the sign after one of the loops in a pair changes the sign (direction) of the flow $\boldsymbol{\Phi}_{i}$.

For a single bubble of the symmetric phase the flow of the helicity density through the surface (6) preserves the value (17). Moreover, for the same $n<0$ this flow does not change the negative sign after the direction of the flow is changed, $\mathbf{n}_{Y} \rightarrow-\mathbf{n}_{Y}=\hat{\mathbf{e}}_{r}=(1,0,0)$. This well corresponds to the meaning of the problem: magnetic helicity of the Maxwellian field rises, unless helicity of the hypermagnetic field inside the bubble goes down.
[1] D. Grasso and H. R. Rubinstein, Phys. Rept. 348 (2001).

[2] M. Giovannini, Int. J. Mod. Phys. D 13 (2004) 391.

[3] A. Brandenburg and K. Subramanian, Phys. Rep. 417, 1 (2005).

[4] V.B. Semikoz and D. D. Sokoloff, Phys. Rev. Lett. 92, 131301 (2004).

[5] V. B. Semikoz and J. W. F. Valle, JHEP 03, 67 (2008).

[6] T.W.B. Kibble and A. Vilenkin, Phys. Rev. D 52, 679 (1995); J. Ahonen, K. Enqvist, Phys. Rev. D 57, 664 (1998).

[7] E. Priest, T. Forbes, Magnetic reconnection; MHD theory and applications, Cambridge University Press, (2000)
8.5.2 (chapter 8, section 5, subsection 2).

[8] V. B. Semikoz, D. D. Sokoloff and J. W. F. Valle, Phys. Rev. D 80, 083510 (2009).

[9] P.M. Akhmet'ev, V.B. Semikoz and D.D. Sokoloff, Pis'ma ZHETF 91, 233 (2010) (in Russian, to be translated in JETP Letters).

[10] M. Giovannini, Phys. Rev. D 61, 063502 (2000).

[11] V.B. Semikoz and D. D. Sokoloff, Astronomy \& Astrophysics 433, L53 (2005); V. B. Semikoz and D. D. Sokoloff, Int. J. Mod. Phys. D 14, 1839 (2005).

[12] T. Vachaspati, Phys. Rev. Lett., 87, 251302 (2001). 